\title{
Investigación y publicaciones de estudiantes de grado y posgrado
}

German Moncada Godoy*

El presente artículo se enfoca en el estado actual de la formación en investigación en el pregrado y el posgrado de la UNAH. Para ello se recogen los antecedentes de la experiencia de la década pasada, se describe los factores curriculares que constituye la estructura sobre la que se articula la formación, se valoran las prácticas educativas para el desarrollo de capacidades investigativas y finalmente se sostiene la hipótesis de que las limitaciones que hay en este proceso educativo es que no se alcanzan a realizar la etapa de divulgación y publicación. Se especula que solo si se llega a este punto es que realmente los estudiantes desarrollarán las capacidades de producción científica.

Desde que Humboldt formulara la relevancia del vínculo docencia-investigación para las universidades "modernas", reclamando para éstas las tareas de investigación científica, que hasta ese entonces venían siendo desarrolladas por academias y sociedades especializadas, la actividad investigadora se ha constituido en un reto permanente para la institución universitaria. Se asumió desde entonces que la investigación científica y tecnológica, es un elemento de primer orden en el proceso de desarrollo de las sociedades, que no debe estar ausente de las tareas universitarias.

Las tendencias actuales indican que las universidades tienen que reevaluar el papel de la investigación, porque en el nuevo escenario de la sociedad del conocimiento se demanda el papel activo de la ciencia y tecnología en el proceso de desarrollo de los países. Tal como fue planteado en la última Conferencia Mundial sobre la Educación Superior (UNESCO, 2009).

"En ningún otro momento de la historia ha sido más importante que ahora la inversión en los estudios superiores, por su condición de fuerza primordial para la construcción de sociedades del conocimiento integradoras y diversas, y para fomentar la investigación, la innovación y la creatividad. La experiencia del decenio pasado demuestra que la investigación contribuye a erradicar la pobreza, a fomentar el desarrollo sostenible y a adelantar en la consecución de los objetivos de desarrollo acordados en el plano internacional, entre otros los Objetivos de

Universidad Nacional Autónoma de Honduras, Facultad de Ciencias Sociales. Escuela de Psicología. moncadapaz@gmail.com 
Desarrollo del Milenio (ODM) y de la Educación para Todos (EPT)" (UNESCO, 2009).

La concepción de universidad moderna está ligada con la investigación que se realiza, la cual sin duda adquiere su máximo nivel en el posgrado. Sin embargo, para lograr esa investigación avanzada es necesario contar con una cultura de investigación la cual se inicia en el pregrado. Es en este nivel en donde se forma 0 deforma el espíritu investigativo, fomentando en el estudiantado su mentalidad crítica, su interés por la indagación, el cuestionamiento permanente y la posibilidad de crear y recrear el conocimiento (Calderón, 2005).

Dentro del contexto universitario, es necesario distinguir entre investigación científica, Strictu Sensu, que es aquella que mueve la frontera del conocimiento y es reconocida y validada por la comunidad académica, y la investigación formativa que tiene como finalidad el desarrollo de capacidades para hacer ciencia (Restrepo, 2002).

En este artículo se aborda los procesos educativos de investigación formativa, que se han configurado en la UNAH, mostrando las debilidades y limitaciones de las que adolece y que muestran el escaso impacto que tienen. Al mismo tiempo, se propone que el eslabón más débil de la formación en investigación está en la fase final, que es la divulgación y/o publicación de los productos alcanzados; se establece que si se logra publicar, se impulsará el perfil de las capacidades de investigación de los estudiantes en proceso de formación en el pre y posgrado.

\section{La sombra del pasado reciente}

Es posible que antes se hiciera más investigación que ahora; se plantea la duda ya que hasta 1989 existía en la UNAH como requisito de graduación, la tesis; durante mucho tiempo se acumularon estos productos en los anaqueles de bibliotecas y centros de documentación. Esta exigencia promovió dentro de los departamentos una estructura académica con capacidades para guiar y desarrollar la investigación, pero en vista de que este proceso se había convertido en un cuello de botella que afectaba la eficiencia terminal, se tomó la decisión se suprimir este requisito.

Los resultados de la medida fueron satisfactorios, ya que se vio que la dificultad que había para graduarse era la incapacidad de los estudiantes de hacer la investigación. A partir de este momento en la UNAH y en el conjunto del sistema educativo superior se le restó importancia a la investigación, ya que no era 
necesario que se enseñara este tipo de contenido y quedó a discreción de cada carrera decidir si se enseñaba o no. Fue hasta el año de 2008, que en el contexto de la cuarta reforma, que se volvió a colocar como requisito de finalización que los estudiantes tomaran un seminario de investigación, proponiendo resolver el vacío que se había dejado. Es evidente que esta medida es muy tibia para asegurar que se desarrollen las competencias investigativas.

Por otra parte, las últimas tres décadas han sido un periodo en el que los posgrados han experimentado la expansión de su oferta, la diversificación de los programas y el aumento de la matrícula. De hecho se ha tornado en una de las principales tendencias del sistema educativo superior. No obstante, estos programas de formación al más alto nivel experimentan un grave problema de eficiencia terminal, en el que de nuevo aparece el fantasma de la no graduación de los estudiantes, que al no poder cumplir con el requisito de hacer la tesis, se quedan frustrados de culminar los estudios.

Dados estos avatares, cabe preguntarse si la universidad ¿debería renunciar a formar a sus estudiantes en investigación? Parece una obviedad decir que no, sin embargo, hay síntomas de que ésta es una tentación que asalta a algunos gestores universitarios; amenaza que de momento no termina de consumarse. Para responder a esta interrogante apropiadamente hay que hacer las siguientes consideraciones: primero, la Constitución de la República, en el artículo 160, exige a la UNAH a que contribuya a la investigación científica, humanística y tecnológica; segundo, en el artículo 3 de la Ley Orgánica de la UNAH se plantea que uno de los objetivos de la institución es promover, desarrollar, innovar y difundir la investigación científica, humanística y tecnológica. Dado que en sus fundamentos legales está el mandato de que tiene que contribuir a la investigación no puede dejar esta actividad, más bien, debe recuperar espacios formativos para lograr este objetivo.

Más allá de su obligación legal, persiste la duda cartesiana de porqué es que hay que formar en investigación y qué se gana con estas competencias y por qué simplemente no formar a los profesionales para que sepan desempeñar un puesto de trabajo al más alto nivel. Esta representación gravita en las mentalidades de estudiantes y docentes que no terminan de entender el valor de la investigación y se materializa en anodinas propuestas de planes de estudios con que se cuenta.

\section{¿Qué expectativas se tienen de la investigación en el currículo?}

Teniendo en cuenta que lo que se espera debe estar claro en la propuesta curricular existente en el pre y posgrado, a continuación se valora lo que se está ofreciendo en 
los espacios formativos garantiza que es posible alcanzar las competencias mínimas de investigación. En este sentido se contabiliza la cantidad de contenido de investigación en los planes de estudio, el tipo de competencias propuestas y los discursos que se hacen en los planes que se establecen como marco de operación.

\section{El target de la formación investigativa en el pre y posgrado}

Retomando el sentido último de la obligación de la universidad de formar en investigación, hay que decir que el pensamiento científico posibilita la resolución de problemas de forma sistemática, promueve actitudes proclives a la investigación científica y ayuda a los profesionales a una mejor inserción. La universidad no puede enseñarlo todo, por eso a los estudiantes hay que "darles la caña de pescar", es decir, una herramienta que les permita aprender a aprender. En un contexto en el que la innovación es algo cotidiano en la que el conocimiento se duplica cada cinco años, y cada seis meses la tecnología es obsoleta, se requiere de profesionales con una nueva mentalidad científica capaz de hacerle frente a este desafío.

En el posgrado la meta se profundiza y se aspira a que el estudiante adquiera las habilidades para poder producir conocimiento. En la maestría se espera habilitarlo para hacer investigación, pero para ello tiene que demostrar que es capaz de seguir un proceso sistemático de producción de conocimiento. El doctorado, por su parte, sí exige la producción de un conocimiento nuevo. No obstante la eficiencia terminal en los posgrados muestra que ello no se está logrado, ni tampoco en el doctorado. Son las especialidades médicas las únicas que han podido dar el ejemplo de logros en producción investigativa. Es ésta la pista que habría que seguir, en este caso el punto de llegada es producir un artículo publicable en una revista científica y no la pesada tesis de 100 a 200 páginas.

La meta educativa ha sido bautizada aquí con el nombre de investigación formativa, ya que esta tiene que ver con dar forma, y estructurar algo a lo largo del proceso. Según Restrepo, (2002) la investigación formativa busca formar a través de actividades propias de la investigación,aunque éstas no logren tener resultados científicos No hay que sobre estimar la investigación en este nivel pensando que la universidad puede dar la cara en materia de ciencia con los trabajos que hagan los estudiantes, esto es desnaturalizar el propósito formativo que tiene este nivel; tampoco hay que subestimarla, haciendo cualquier cosa, hay que ponerla en su lugar, como parte de un proceso formativo, con una finalidad pedagógica. 


\section{La investigación como un apéndice del currículo}

En un estudio realizado por Ledezma (2009), se estableció que en el pregrado de la UNAH hay un $4 \%$ de asignaturas de investigación, lo cual es un peso bastante escaso, no obstante la importancia relativa es variable, ya que hay un $19 \%$ de las carreras que no tienen asignaturas de métodos de investigación. Hay carreras que no valoran absolutamente la investigación, que son la mayoría, y carreras que tienen una buena dosis de clases con estos contenidos. Por su parte, los posgrados tienen en promedio un $22 \%$ de asignaturas del área de investigación, pero el peso asignado es variable. Existen programas que no cuentan con este tipo de asignaturas y otros que tienen 8 asignaturas de 19 posibles. Paradójicamente "más no quiere decir mejor", como lo demostró un estudio en el que se relacionó el número de créditos con la eficiencia terminal observándose que no hay correlación y que por el contrario los programas con más cursos de investigación eran menos eficientes (Moncada y Flores, 2010). La inclinación a la docencia se evidencia en la cantidad de cursos de consumo de conocimiento y no de producción de conocimiento, lo que en definitiva relega la investigación a un lugar secundario.

\section{Apología de la investigación científica en el discurso académico}

El discurso que gravita en relación a la investigación frecuentemente es una retórica en la que aparece la formación científica como una prioridad. La planificación de cualquier carrera universitaria tiene como eje la investigación aplicada a sus respectivas disciplinas. Este contenido se considera clave en los discursos que se hacen sobre la formación al más alto nivel. Detrás de este esfuerzo de reivindicar la investigación es que entre más se plantea, más prestigio se tiene.

No obstante, esta supuesta prioridad no se ha traducido en la realización de estudios científicos en la escala que plantean los discursos; de hecho, la realidad parece ir en otra dirección; tal como se demostró cuando se decidió eliminar las tesis como requisito de graduación. En otras palabras, la investigación en la práctica era un problema y había que sacudírselo. Esta misma situación resurge actualmente en los posgrados, en los que hay cada vez más egresados, pero hay muy pocos graduados. La dificultad para hacer un trabajo de tesis es la muestra definitiva que no se está logrando impacto en la formación científica.

Este resultado es producto de un contexto más general, en el que la investigación continua siendo la cenicienta en la universidad, es decir una cuestión de la cultura, los hábitos y las costumbres que se tienen. A pesar de los esfuerzos destacados que viene haciendo la UNAH, a través de la Dirección de Investigación Científica, queda 
mucho camino por recorrer, por lo que esta actividad se convierte en un desafío y tarea pendiente. No se trata solo de decisiones al más alto nivel, se trata también de que los docentes cotidianamente en el aula desarrollen buenas prácticas de búsqueda e indagación sistemática.

\section{Competencias básicas para la investigación}

En pregrado el objetivo no es formar investigadores, pero si le corresponde formar profesionales con actitudes positivas hacia la investigación, de manera que se convierta en sus usuarios y la adopten como una forma habitual de afrontar problemas cotidianos.

Cuando se habla de investigación en el contexto universitario se hace referencia no sólo a hacer investigación, sino más bien prácticas, habilidades y aptitudes para el quehacer investigativo y, lo más importante, para la vida.

En este sentido las competencias que es necesario fomentar, se refieren a habilidades básicas como la lectura comprensiva y crítica, la escritura, el cálculo, la computación y el manejo de una segunda lengua; elementos difíciles de encontrar en los estudiantes de pregrado y a veces en posgrado. Así mismo se requiere el perfeccionamiento de habilidades de abstracción, pensamiento sistemático, experimentación y colaboración. También existe la necesidad de fomentar habilidades específicas como el reconocimiento de la especificidad epistemológica, metodológica e instrumental de las disciplinas y profesiones y el uso de herramientas especializadas por parte de los futuros profesionales (Guerrero, 2007).

Una competencia que hay que lograr desarrollar en los futuros estudiantes es que puedan redactar un paper, en ello es importante la guía y supervisión adecuada, así como una gran dosis de perseverancia para leer, pensar, revisar errores, re-escribir, y elaborar ideas una y otra vez. Se ha dicho que somos una cultura oral y no de letras impresas, por lo que va a costar un poco romper con este componente atávico.

\section{Prácticas pedagógicas en la formación investigativa en el pre y posgrado}

Como se ha visto, en los planes curriculares existe aunque en forma reducida la investigación, la cuestión es ¿qué hay de la ejecución?, ¿cómo se enseña? En este sentido se pueden realizar varios apuntes, en primer lugar, de como se reduce la investigación al método, del uso del texto como la versión de la verdad sobre el 
tema, del aprender a investigar investigando y del fuerte componente ético que debe acompañar la investigación.

\section{Reduccionismo metodológico en la enseñanza de la investigación}

Existe la tendencia, en el ámbito académico, de reducir la investigación a la aplicación de la metodología de la investigación, al uso de ciertos aspectos del método científico y a pensar que la investigación científica es el método. En este sentido cuando Bunge (1989) establece que no hay reglas, que reglamentar la ciencia fue una preocupación en el pasado (Bacon, Descartes, Durkheim), hoy se ha desterrado este intento ya que hemos arribado a la conciencia de que estos siempre serán susceptibles de perfección.

No obstante, las asignaturas de métodos de investigación suelen ser espacios donde se plantea el proceso como si fueran reglas y normas. En este sentido Ruiz \& Torres (2005), señalan que la asignatura de investigación no ha contribuido a la formación de investigadores y lo atribuyen a las tendencias didácticas y a los supuestos que soportan el ejercicio de la docencia centrado en el enfoque tradicional transmisionista.

La enseñanza de la investigación, tal como se presenta en el sistema educativo actual, es lineal. No estimula a los estudiantes a aventurarse a producir conocimiento, ni a apropiárselo de manera creativa en la solución de los problemas.

Esta crítica a la enseñanza de la investigación es compartida por Cerda (2007), cuando dice que la enseñanza de la investigación, es más informativa que formativa. La mayoría de programas de pregrado tienen varios niveles de formación en metodología de la investigación, estadística, seminarios de redacción científica, informática, entre otros, en los cuales la investigación se presenta a manera de recetas, no como algo susceptible de ser comprendido, mejorado o completado. En la mayoría de los casos no se facilita la construcción de espacios para integrar todos estos saberes de manera clara, productiva y amena para el estudiante y para el mismo docente.

\section{Sampieri o el libro sagrado}

En la práctica pedagógica se requiere el apoyo de materiales educativos especialmente textos. El libro más usado en el campo de la investigación es el denominado libro de "Sampieri". Es un texto muy accesible en el mercado, que ofrece un panorama de los pasos del proceso de investigación, diseñado para ser 
usado en varias disciplinas de las ciencias sociales. Su uso, en ocasiones traslada la sensación de estar frente a un contenido doctrinario de lo que es la investigación, ya que da la impresión de que da una receta que siguiéndola se puede llegar a hacer ciencia. Los alumnos a veces argumentan diciendo "Sampieri dice". A pesar de las limitaciones de libros en el mercado hondureño, hay que señalar que hay disponible otra literatura y otros recursos que pueden ser significativos para comprender la investigación en cada disciplina, que no se están usando, tal es el caso de blogs de investigación cualitativa y cuantitativa. La biblioteca virtual de la UNAH tiene disponible varias bases de datos que poseen Journals de investigación del más alto nivel, en texto completo, contiene además una importante colección de e-books y como si no fuera suficiente, hay disponibles bases de datos gratuitas como Scirus, Redalyc, Scielo, que tienen una buena cantidad de revistas científicas gratuitas y de un gran nivel. Ya no es posible esgrimir la frase no hay bibliografía. Ahora el reto es como gestionar toda esta información disponible.

\section{Aprender a investigar investigando}

Para un abordaje exitoso de la investigación dentro del currículo, hay que tener presente que las competencias en investigación no se logran únicamente mediante un esfuerzo de pedagogía de la investigación tradicional. Más bien, se trata de construir una cultura de la investigación que permita formar profesionales más críticos, con capacidad de sistematizar, analizar y capitalizar la experiencia. Tal y como lo menciona Campos y Chinchilla (2009) el desarrollo de las competencias investigativas requiere la construcción de una cultura de investigación. Con disciplina, empeño y una buena formación, es posible que muchos estudiantes puedan llegar a ser investigadores de calidad.

\section{La ética de la investigación}

Es frecuente encontrar que los estudiantes cometen varias violaciones al código de ética de la investigación, por ejemplo el plagio, también en la recolección de los datos suelen haber alteraciones. Los componentes actitudinales y éticos vinculados a la investigación suelen subestimarse, incluso se tolera este tipo de acción, lo que explica que por omisión hay cierta complicidad que ha provocado que se mantengan este tipo de acciones. Los estudiantes ven que se puede hacer sin correr ningún riesgo, ya que se sabe que no hay mayores consecuencias.

En resumen, una buena práctica en la enseñanza de la investigación se vincula a no reducir el proceso a unas etapas, desarticuladas y fragmentadas, pues ese reduccinismo crea una perspectiva que hace perder valor a la investigación. Se 
requiere para cambiar ésto, llegar al final del proceso a la divulgación de los resultados; ello va a generar mayor compromiso replanteando e innovando las prácticas de enseñanza.

\section{"Publish or perish"}

Hasta aquí se ha expuesto la problemática de la enseñanza y el aprendizaje de la investigación y se ha sugerido la importancia de la publicación. A continuación se plantea la hipótesis de que la divulgación de las investigaciones de los estudiantes y las tesis de posgrado pueden cambiar el actual estado de declive que experimentan los procesos formativos. La sentencia "publicar o perecer" hace referencia a la necesidad de publicar los resultados de investigación para poder mantenerse en el mundo académico; como un criterio de supervivencia ya que la investigación que no se publica no existe. En otras palabras, lo de hacer investigación es una dicotomía que se puede dictaminar con la frase "está publicado o no".

\section{Por qué es importante publicar desde el pregrado y el posgrado}

Porque terminar el proceso de investigación es llegar a la divulgación; y al hacerlo se gana y adquiere experiencia. La habilidad de publicar se desarrolla con la práctica. Si se publica una vez, es probable que lo siga haciendo ya que se forma el hábito; si se ha gastado esfuerzo en hacerlo, lo consecuente es publicarlo, puede servir de carta de presentación para el futuro, ya que otros investigadores contrastan, refutan, repiten o mejoran lo que se encuentra.

Hay que decir que en otros contextos hay revistas científicas indexadas de alto impacto que son hechas por estudiantes. Como por ejemplo la Asociación Nacional Científica de Estudiantes de Medicina de Chile (ANACEM) formada desde 1994 en la ciudad de Concepción, la cual ha tenido desde sus orígenes como principal objetivo promover, apoyar y difundir el desarrollo de la ciencia e investigación médica desde el pregrado, en pro de contribuir a la salud del país. Esta publicación está abierta a todos los estudiantes del área de la salud y profesionales del ámbito, sin distinción ni predilección por carrera o universidad, con la condicionante de que en el equipo de trabajo exista al menos un estudiante de la carrera de Medicina. Está indexada en Ebsco, Latindex y Lilacs.

También, vale la pena decir que es frecuente que en las publicaciones que se realizan en academias muy desarrolladas sean contribuciones en las que colaboran docentes y estudiantes. Es por esta razón, que sería deseable embarcarse en el proyecto de promover las publicaciones en distintos formatos como por ejemplo congresos, seminarios, blogs, páginas de Internet, posters y trifolios. 
La Revista Portal de la Ciencia de la Dirección de Investigación Científica, es una iniciativa orientada a ofrecer a los estudiantes una oportunidad de colocar sus trabajos cuando éstos sean de calidad. En Honduras es una ventana que se abre para alcanzar el objetivo de tener medios de difusión de los productos investigativos.

La publicación es parte del proceso de investigación. A pesar de que suele verse como algo distinto e incluso suele no terminarse el proceso, en algunos espacios educativos se llega hasta formular un plan de investigación. En otros se llega a un informe, pero en casi ningún caso se considera que hay que llegar a estructurar un artículo y publicarlo en algun medio. No obstante hay que tomar conciencia que solo si se publica se cierra el ciclo de la investigación, de lo contrario queda incompleto. En algunos casos excepcionales existe el potencial para que sean conocimientos que alcancen un nivel competitivo que puede ser publicado en revistas científicas y que en consecuencia aporte algo al acervo científico universal. Ésta no debe ser la motivación, sí ocurre, bienvenido, pero no es el objetivo, ya que los estudiantes están en proceso de formación, por lo que pueden publicar y empezar a tener experiencias, foguearse, someterse a la crítica, que es la que les permitirá ir mejorando y cumplir con el sueño de ser los profesionales que necesita Honduras.

\section{BIBLIOGRAFÍA}

- Bunge, M. (1989). La Investigación Científica. Su Estrategia y su Filosofía. Barcelona:Ariel.

- Calderón, G. (2005). Aprender a investigar investigando: Errores más frecuentes en el proceso investigativo y como evitarlos. Manizales. Universidad Nacional de Colombia.

- Campos, J. \& Chinchilla, A. (2009). Reflexiones acerca de los desafíos en la formación de competencias para la investigación en educación superior. Revista Electrónica publicada por el Instituto de Investigación en Educación. 9. (2). 1-20. http://www.latindex.ucr.ac.cr/aie-2009-2/aie-2009-9-23.pdf.

- Cerda, H. (2007). La investigación formativa en el aula: la pedagogía como investigación. Bogotá. Magisterio.

- Guerrero, M. (2007). formación de habilidades para la investigación desde el pregrado. Acta colombiana de psicología. 10 (2). 190-192

- Ledezma, R. (2009). Diagnóstico de la investigación en el pregrado. Tegucigalpa. DICU.

- Moncada, G \& Flores, M. (2010). La Investigación Científica en los Postgrados de la UNAH. Tegucigalpa. DICU. 
- Restrepo, B. (2002). Conceptos y aplicaciones de la investigación formativa y criterios para evaluarla investigación en sentido estricto. Bogotá. CNA.

- Ruiz, C., \& Torres, V. (2005). La enseñanza de la investigación en la universidad: el caso de una universidad pública venezolana. Investigación \& Postgrado, 20(2).

- UNESCO (2009). Conferencia Mundial sobre la Educación Superior - 2009: La nueva dinámica de la educación superior y la investigación para el cambio social yel desarrollo. París. 